Article

\title{
Temperature Compensated Wide-Range Micro Pressure Sensor with Polyimide Anticorrosive Coating for Harsh Environment Applications
}

\author{
Mengru Jiao ${ }^{1}$, Minghao Wang ${ }^{1,2, *} \mathbb{D}$, Ye Fan ${ }^{1}$, Bangbang Guo ${ }^{1}$, Bowen $\mathrm{Ji}^{3}{ }^{\mathbb{D}}$, Yuhua Cheng ${ }^{1,2, *}$ \\ and Gaofeng Wang ${ }^{1, *(D)}$ \\ 1 MOE Engineering Research Center of Smart Microsensors and Microsystems, School of Electronics \& \\ Information, Hangzhou Dianzi University, Hangzhou 310018, China; jmr@hdu.edu.cn (M.J.); \\ 16041701@hdu.edu.cn (Y.F.); bbguo@hdu.edu.cn (B.G.) \\ 2 Wenzhou Institute of Hangzhou Dianzi University, Wenzhou 325038, China \\ 3 The Unmanned System Research Institute, Northwestern Polytechnical University, Xi'an 710060, China; \\ bwji@nwpu.edu.cn \\ * Correspondence: mhwang@hdu.edu.cn (M.W.); chengyh@hdu.edu.cn (Y.C.); gaofeng@hdu.edu.cn (G.W.)
}

check for updates

Citation: Jiao, M.; Wang, M.; Fan, Y.; Guo, B.; Ji, B.; Cheng, Y.; Wang, G. Temperature Compensated Wide-Range Micro Pressure Sensor with Polyimide Anticorrosive Coating for Harsh Environment Applications. Appl. Sci. 2021, 11, 9012. https:// doi.org/10.3390/app11199012

Academic Editor: Dimitrios-Nikolaos Pagonis

Received: 18 July 2021

Accepted: 24 September 2021

Published: 28 September 2021

Publisher's Note: MDPI stays neutral with regard to jurisdictional claims in published maps and institutional affiliations.

Copyright: (c) 2021 by the authors. Licensee MDPI, Basel, Switzerland. This article is an open access article distributed under the terms and conditions of the Creative Commons Attribution (CC BY) license (https:// creativecommons.org/licenses/by/ $4.0 /)$.

\begin{abstract}
In this work, a MEMS piezoresistive micro pressure sensor $(1.5 \times 1.5 \times 0.82 \mathrm{~mm})$ is designed and fabricated with SOI-based micromachining technology and assembled using anodic bonding technology. In order to optimize the linearity and sensitivity over a wide effective pressure range $(0-5 \mathrm{MPa})$ and temperature range $\left(25-125^{\circ} \mathrm{C}\right)$, the diaphragm thickness and the insulation of piezoresistors are precisely controlled by an optimized micromachining process. The consistency of the four piezoresistors is greatly improved by optimizing the structure of the ohmic contact pads. Furthermore, the probability of piezoresistive breakdown during anodic bonding is greatly reduced by conducting the top and bottom silicon of the SOI. At room temperature, the pressure sensor with $40 \mu \mathrm{m}$ diaphragm demonstrates reliable linearity $(0.48 \%$ F.S.) and sensitivity $(33.04 \mathrm{mV} / \mathrm{MPa})$ over a wide pressure range of $0-5.0 \mathrm{MPa}$. In addition, a polyimide protection layer is fabricated on the top surface of the sensor to prevent it from corrosion by a moist marine environment. To overcome the linearity drift due to temperature variation in practice, a digital temperature compensation system is developed for the pressure sensor, which shows a maximum error of $0.43 \%$ F.S. in a temperature range of $25-125^{\circ} \mathrm{C}$.
\end{abstract}

Keywords: temperature compensation; wide-range; pressure sensor; polyimide anticorrosive coating; harsh environment

\section{Introduction}

The piezoresistive effect of a semiconductor was first discovered in the 1950s, which is the theoretical basis of most pressure sensors [1-3]. In addition to piezoresistive sensors, several different transduction mechanisms have been applied to convert pressures or strains to signals, such as inductance, capacitance, piezoelectricity, and resonance, which can be detected on circuitry [4,5]. Among these designs, piezoresistive sensors are generally accepted due to their simple construction and low energy consumption [6]. The micro pressure sensors have been greatly developed and played a pivotal role in the automobile industry, aerospace industry, and medical equipment with the promotion of MEMS technology [7-11]. In marine engineering, pressure sensors are commonly used in applications involving high pressure up to several megapascal $(\mathrm{MPa})$, high temperature up to several hundred degrees Celsius, and moist external conditions. However, some intrinsic pitfalls of the silicon piezoresistive pressure sensor, such as inadequate performance in harsh environment, e.g., high pressure or high temperature, and output drift due to temperature variation, have limited its application in marine settings. 
In order to improve the performance of the micro piezoresistive pressure sensor, numerous studies have focused on optimization of structure, fabrication, and encapsulation. Dong et al. [12] introduced a monolithic composite MEMS pressure sensor with an effective range of $450 \mathrm{kPa}$. Nag et al. [13] modified the structure by integrating rod beams in a silicon diaphragm. A pressure range of 0-689.5 kPa was achieved. Sheeparamatti et al. [14] fabricated two micro pressure sensors based on polysilicon on insulator (PolySOI) and amorphous silicon on insulator (a-SOI). These two sensors provided a pressure range of 0-1 MPa. Alcheikh et al. [15] presented the fabrication and electromechanical characterization of silicon-based 3D force micro sensors with piezoresistive gauges. The high sensitivity, the high linearity, and the low hysteresis of the sensors submitted to a normal force are obtained. Sujit et al. [16] incorporated FEM (finite element method) to give suggestions on the selection of parameters for the piezoresistors, such as shape and the position on the sensitive diaphragm.

To prevent micro sensors from being damaged by external environment, most pressure sensors are encapsulated by silicone rubber. However, a large deformation of silicone rubber under a high pressure could lead to a fracture of bonding wires. Previous published work $[17,18]$ has presented an anticorrosive PDMS (polydimethylsiloxane) coating to prevent the conductive layer of a strain sensor from being damaged by corrosive external environment. In addition, Won et al. [19] spun polyimide film onto the electrode of the sensor to protect it from the humid environment. However, hardly any previous research has focused on the performance and protection of the micro sensors under a harsh environment, such as high pressure, high temperature, or moist corrosive electrolyte environment, which will lead to a shortening of the micro sensor life. Therefore, our work aims at resolving the contradiction between the output performance and stability with a miniature size in harsh environment.

Previous research has established two approaches to treat nonlinearity temperature drift. The first approach is to use an analog compensation circuit, such as the external thermistor. Aryafar et al. [20] presented a passive technique for temperature compensation of a silicon pressure sensor based on extra polysilicon resistors with negative temperature coefficient of resistivity. The second approach is to use a digital compensation system with the combination of electronic circuits and specific software. However, the first approach has intrinsic pitfalls such as poor reliability, low accuracy, and high design difficulty [21]. Concerning the second approach, neural network has recently been applied. Yang et al. proposed an artificial neural network (ANN) [22] to establish a temperature shift compensated model. Zhou et al. developed an extreme learning machine (ELM) to calibrate the pressure shift [23]. Neural networks, however, require a long training time and a large amount of calculation, which is a barrier for marine engineering applications. Therefore, our work proposes a low-cost pressure measurement system with temperature compensation based on embedded technology and rational polynomial fitting to reduce the cost of temperature compensation and increase the flexibility of the compensation system.

In this work, a MEMS piezoresistive pressure sensor with ideal performance over a wide effective range by optimizing the micromachining process is designed, simulated, fabricated, and tested. Firstly, the optimization greatly enhances the performance and the stability of the pressure sensor under harsh environment such as high temperature and pressure. Secondly, a novel polyimide anticorrosive layer is fabricated by polyimide film on the top of the micro sensor to prevent it from corrosion by an electrolyte such as seawater. Finally, to compensate the temperature drift, a digital low-cost temperature compensation system is built for this micro pressure sensor.

\section{Design and Fabrication}

\subsection{Design and Fabrication of the Micro Sensor}

In general, a piezoresistive pressure sensor has a sensing diaphragm [24-27]. For piezoresistive sensors, the sensitive diaphragms above the vacuum cavities are pressured and then generate stress. The variation of piezoresistors caused by the applied pressure is 
transferred into voltage due to the piezoresistive effect [28,29]. Therefore, the performance of a piezoresistive sensor depends largely on the pressure sensitive diaphragm. Firstly, the dimensions of the pressure sensor's diaphragm were designed based on previous published work [12]. Then, the thickness of the diaphragm was determined by theoretical calculation from Expression (1), where $P_{\text {burst }}$ is five times the maximum applied pressure, $\sigma_{\text {fracture }}$ is the maximum stress of the diaphragm, $A$ is the area of the diaphragm, $h$ is its thickness, and $v$ is the Poisson's ratio. The calculated minimum thickness is $26.87 \mu \mathrm{m}$. Therefore, the simulation was done in COMSOL Multiphysics FEA (finite element analysis) software for a thickness of more than $30 \mu \mathrm{m}$. Based on this, the pressure sensor models have an n-type silicon diaphragm with the dimensions $900 \times 450 \mu \mathrm{m}$ and thicknesses of 40 and $50 \mu \mathrm{m}$. The von Mises stress induced in the diaphragm with various thicknesses were determined and evaluated with the analytic explanation for a face-loaded pressure of $5 \mathrm{MPa}$. The simulation results obtained from the FEA tool are shown in Figure 1a-d. Along the centerline of the diaphragm, the tensile stress at the diaphragm edge reaches its maximum and the compressive stress in the center of the diaphragm reaches its maximum. This resulted from the silicon diaphragm contracting at the center and stretching at the edges when the pressure was applied uniformly from the top. Therefore, the piezoresistors should be located in these places to increase the sensitivity. By comparing the simulation results of the pressure sensor models with different diaphragm thicknesses, it can be found that the surface stress of the pressure sensor with $40-\mu \mathrm{m}$-thick membrane is larger, indicating that the thinner membrane thickness exhibits the higher sensitivity.

$$
P_{\text {burst }}=\frac{3.4}{1-v^{2}} \times \frac{\sigma_{\text {fracture }} \times h^{2}}{A}
$$

(a)

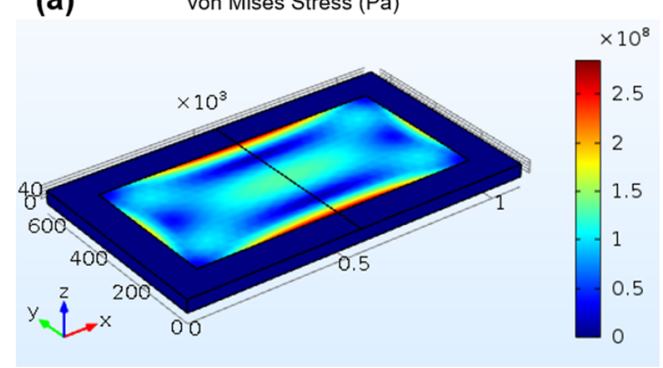

(c)

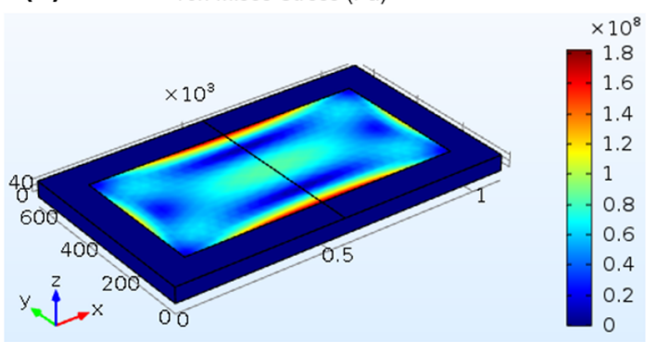

(b)

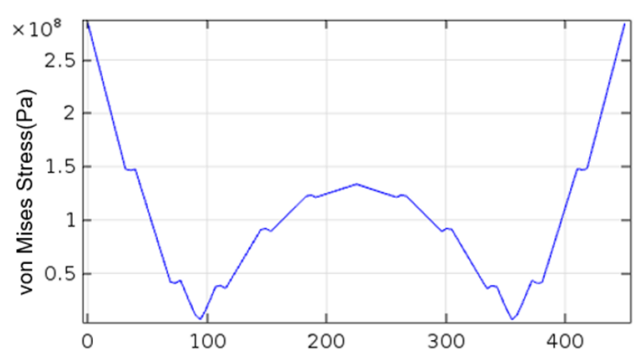

(d)

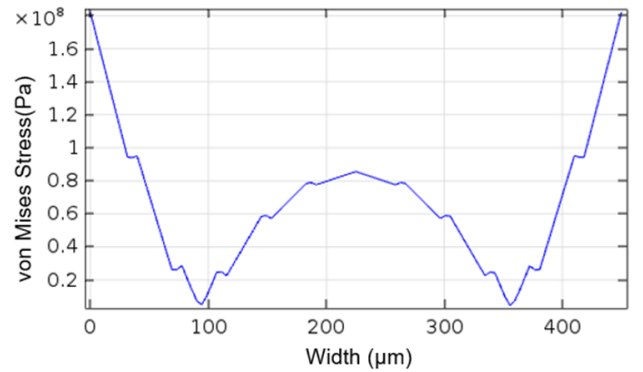

Figure 1. Von Mises stress distribution under finite element analysis: $(\mathbf{a}, \mathbf{c})$ stress distribution under 5 $\mathrm{MPa}$ of face-loaded pressure; $(\mathbf{b}, \mathbf{d})$ transverse stress distribution along the centerline.

The schematic diagram of the proposed silicon piezoresistive pressure sensor is shown in Figure 2. The pressure sensor has a rectangular silicon diaphragm, which is fabricated on the device layer of a SOI wafer [30,31]. By using buried-oxygen-layer etching stop technology [32], the diaphragm thickness of the pressure sensor can be precisely controlled. After one-mask deep reactive ion etching (DRIE), the pressure sensor's rectangular diaphragm is shaped. The thickness of the diaphragm is the same as that of the device layer. The piezoresistors are formed on the silicon diaphragm by boron ion implantation. Four 
piezoresistors are connected to a Wheatstone bridge structure by forming ohmic contact with aluminum wires. The contact area between the aluminum conductor and the heavily doped region of the piezoresistors has a great influence on the resistance value of the four piezoresistors in the Wheatstone bridge. Therefore, the consistency of ohmic contact area should be ensured by improving the accuracy of lithography and etching, and increasing the width of aluminum wire relative to that of piezoresistors. Furthermore, a layer of $\mathrm{SiO}_{2}$ is deposited and patterned on the substrate in the plasma enhanced chemical vapor deposition (PECVD) system, as a passivation layer to improve the reliability of pressure sensors in harsh environments. Dimensions of the diaphragm and the piezoresistors are shown in Figure 2c. The silicon diaphragm has dimensions of $900 \times 450 \times 50 / 40 \mu \mathrm{m}$. Four boron-doped piezoresistors $(250 \times 20 \mu \mathrm{m})$ are arranged in parallel on the diaphragm along the $<110>$ direction and connected with aluminum wire to form a Wheatstone bridge.

(a)

(c)
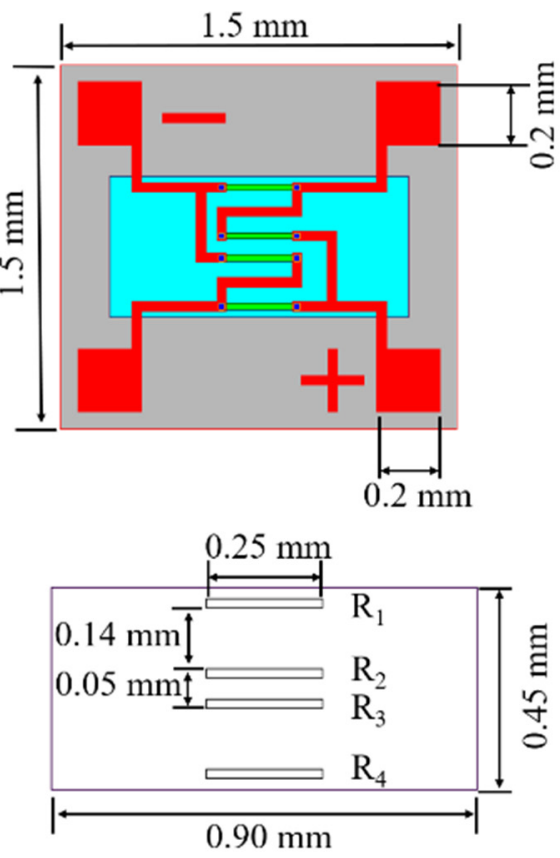

(b)

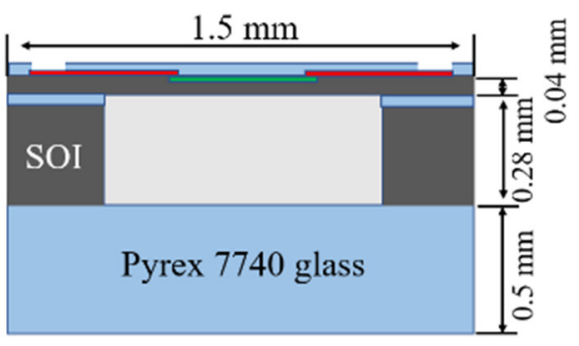

(d)

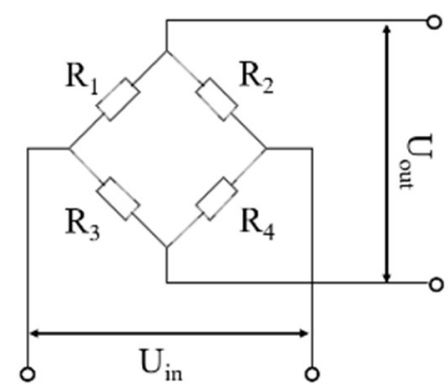

Figure 2. Schematic diagrams of the silicon piezoresistive pressure sensor: (a) top view and (b) crosssection view of the pressure sensor; (c) dimensional parameters; (d) Wheatstone full-bridge of the pressure sensor.

The silicon piezoresistive pressure sensor is fabricated with the micromachining technology and assembled using the anodic bonding technology. As illustrated in Figure 3, the substrate of the pressure sensor is an n-type (100) SOI wafer with a resistivity of 1-10 $\Omega \cdot \mathrm{cm}$. The thicknesses of the silicon device layer, the buried oxide layer, and the handle layer of the SOI are 50/40, 0.5 , and $280 \mu \mathrm{m}$, respectively. The main processes follow: Firstly, the device layer is patterned by photoresist and doped by boron implantation with a doping concentration of $4 \times 10^{14}$ atoms $/ \mathrm{cm}^{2}$ to form piezoresistors (steps 1 and 2). The doped region is then covered by a polyimide film, exposing only the alignment marks. By using the deep reactive ion etching (DRIE) technique, the alignment marks are transferred to the silicon substrate (step 3). Secondly, the contact areas between the $\mathrm{Al}$ wires and the piezoresistors are exposed by patterned photoresist and doped by heavy boron implantation with a doping concentration of $2 \times 10^{15}$ atoms $/ \mathrm{cm}^{2}$ to form the ohmic contact (step 4). Subsequently, a layer of $500 \mathrm{~nm}$-thick silicon oxide is deposited in the PECVD system to form the bottom passivation layer, while the ohmic contact areas are exposed by RIE (steps 5 and 6). Then, a layer of $1 \mu \mathrm{m}$-thick $\mathrm{Al}$ is sputtering deposited and patterned by wet etching to form interconnects and four output pads of the Wheatstone bridge (step 7). 
After that, a layer of $500 \mathrm{~nm}$-thick silicon oxide is deposited in the PECVD system to form the top passivation layer with the output pads exposed by RIE (steps 8 and 9).

(1)

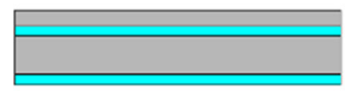

(2)

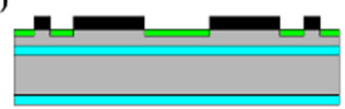

(3)

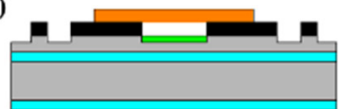

(4)

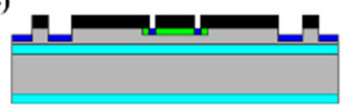

(5)

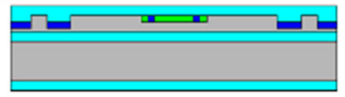

(6)

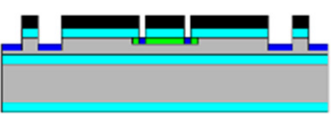

(7)

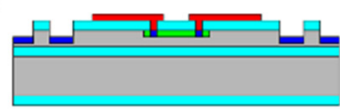

(8)

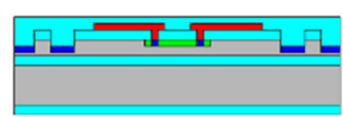

(9)

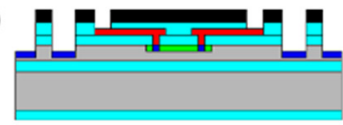

(10)

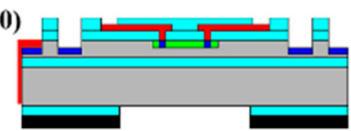

(11)

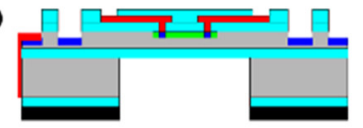

(12)

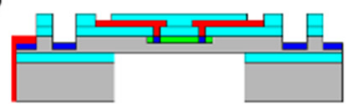

(13)

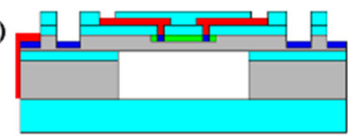

(14)
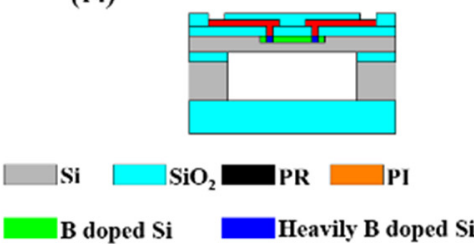

Figure 3. Fabrication process of the silicon piezoresistive pressure sensor.

In order to prevent the pizeoresistors from breakage by the voltage applied during anodic bonding, the passivation layers on the edge of the SOI are also etched to expose the device layer and another $1 \mu \mathrm{m}$-thick $\mathrm{Al}$ layer is sputtered and patterned to connect the device layer and handle layer. Next, the pressure sensor's sensing diaphragm is patterned by double-sided alignment lithography and etched by DRIE and RIE (steps 10, 11, and 12). After polishing by CMP, the handle layer of SOI is bonded to Pyrex7740 glass by anodic bonding with voltage of $1600 \mathrm{~V}$, current of $20 \mathrm{~mA}$, temperature of $300{ }^{\circ} \mathrm{C}$, pressure of $1600 \mathrm{~N}$, and time of $20 \mathrm{~min}$ (step 13). Finally, the sensor dies are released from the SOI wafer by using a dicing saw (step 14).

\subsection{Fabrication of Polyimide(PI) Protection Layer}

To protect the micro sensor from the corrosion of the external environment, such as the electrolytic salty seawater, a protective layer composed of a PI (polyimide) film was developed. Polyimide is a kind of organic polymer material with excellent comprehensive properties, such as better high temperature resistance than PDMS, excellent insulation properties, and stability under electrolyte condition. Meanwhile, polyimide can be well attached on the surface of the devices due to its ductility and flexibility. Therefore, a polyimide film was selected as the protective layer for the micro sensor and for protection of the bonding wires.

After bonding the sensor with packaged PCB by gold wire, polyimide solution of $15 \%$ concentration was placed into a vacuum chamber for $30 \mathrm{~min}$ to remove the air bubbles. Then, the solution was uniformly dripped onto the top surface of the micro pressure sensor. A high-speed rotary coating machine was used to make the polyimide solution form a uniform thin film. The rotation speed, which is an important factor for the thickness of the polyimide film, was set at $500 \mathrm{rpm}$ for $10 \mathrm{~s}$, then $3000 \mathrm{rpm}$ for $30 \mathrm{~s}$. After spinning, the micro sensor with polyimide film was placed in an oven for further solidification. The heating process for the polyimide film was $80^{\circ} \mathrm{C}$ for $10 \mathrm{~min}, 120^{\circ} \mathrm{C}$ for $30 \mathrm{~min}, 150{ }^{\circ} \mathrm{C}$ for $10 \mathrm{~min}, 180^{\circ} \mathrm{C}$ for $10 \mathrm{~min}$, and then $220^{\circ} \mathrm{C}$ for $40 \mathrm{~min}$. Finally, a thin polyimide film with a thickness of $10 \mu \mathrm{m}$ was covered on the top surface of the micro pressure sensor as a protective layer to prevent corrosion by moist air and electrolyte droplets. The device with a $10 \mu \mathrm{m}$-thin PI protection film and the dice itself are shown in Figure 4. 


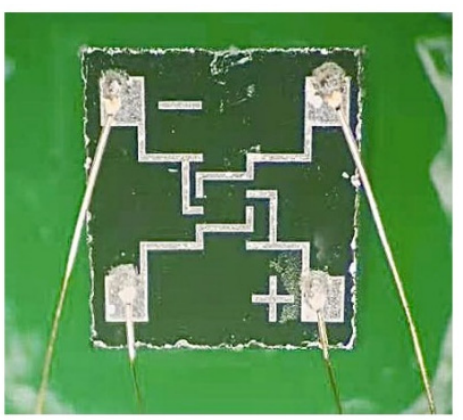

(a)

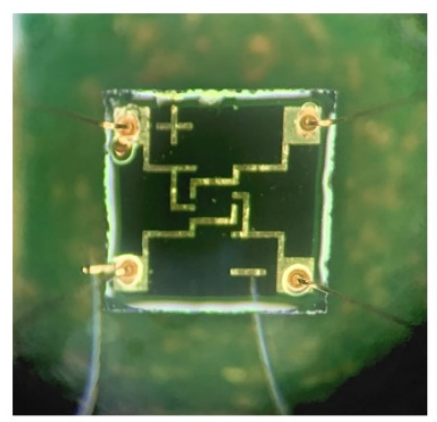

(b)

Figure 4. (a) Micro pressure sensor after bonding without a protection layer. (b) Micro pressure sensor after bonding with a $10 \mu \mathrm{m}$ PI protection layer.

\section{Results and Discussions}

\subsection{Output Characteristics under High Temperature}

The output characteristic of the pressure sensor was measured by a digital pressure test system consisting of a high-precision piston pressure meter, a hot air gun, and a control system. A constant DC voltage of $3.3 \mathrm{~V}$ was applied as the incentive power of the Wheatstone bridge, and the output voltage was captured by a digital multimeter (VC8246B).

To test the output characteristics under different temperature conditions, a piezoresistive pressure sensor with a $50 \mu \mathrm{m}$ sensing diaphragm was pressured from 0 to $3 \mathrm{MPa}$ and the output voltage of the Wheatstone bridge was recorded for every $0.2 \mathrm{MPa}$. The temperature test points were set from 25 to $125^{\circ} \mathrm{C}$ for every $10^{\circ} \mathrm{C}$. The V-P characteristic curve of the pressure sensor under different temperatures is depicted in Figure 5. As shown in Figure 5a, the output voltage exhibits an expected linear correlation in a wide effective pressure range from 0 to $3 \mathrm{MPa}$ and a temperature range from 25 to $125^{\circ} \mathrm{C}$. Sensitivity of the pressure sensor is the ratio of response change to excitation pressure change, and the nonlinearity is the maximum deviation between the actual output of the device and the ideal output curve that changes linearly with the input pressure. Fitting the experimental data with a linear least-square technique, the sensitivity of the pressure sensor was $22.99 \mathrm{mV} / \mathrm{MPa}$ and the nonlinearity was $0.25 \%$ F.S. at $25{ }^{\circ} \mathrm{C}$. Due to the application of SOI wafer, the pressure sensor showed a stable performance with the nonlinearity of $0.31 \%$ F.S. under $125^{\circ} \mathrm{C}$.

(a)

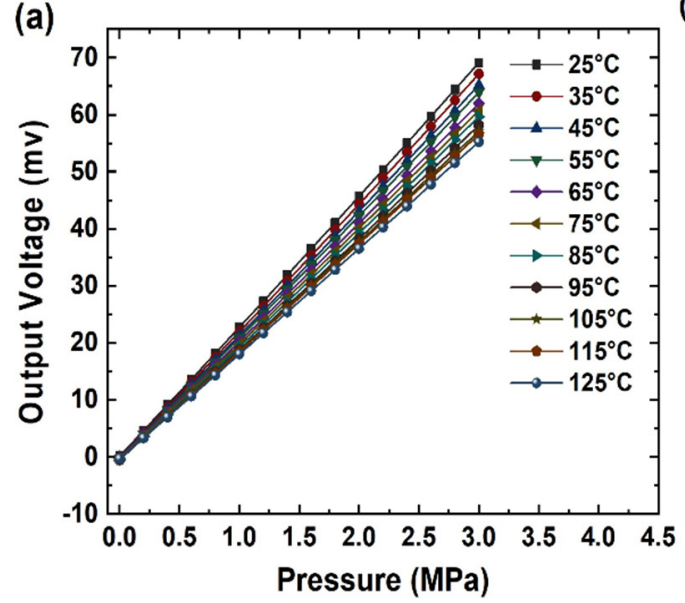

(b)

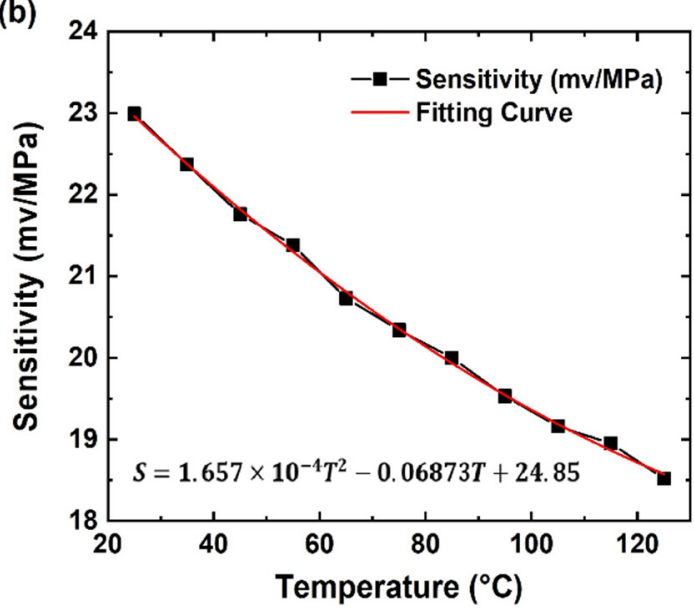

Figure 5. Output characteristics of the silicon piezoresistive pressure sensor: (a) output voltage versus pressure and (b) sensitivity versus temperature. 
The ambient temperature would greatly influence the output characteristics of the pressure sensor. The curve of the sensitivity variation versus the temperature is shown in Figure $5 \mathrm{~b}$. To depict the relation between sensitivity and temperature more accurately, the experimental data is fitted by a two-degree polynomial. The results show that the increase of ambient temperature can still lead to a distinct decline of the sensitivity. This is because the buried oxide layer can only reduce the leakage current of the piezojunction in the handle layer, but not in the device layer of the SOI wafer.

\subsection{Output Characteristics under High Pressure}

In subsequent experiments of pressure measurement range, two devices with different diaphragm thicknesses, 40 and $50 \mu \mathrm{m}$, were tested. The V-P curves of the two devices are presented in Figure 6. As depicted, the device with the thinner diaphragm showed a higher sensitivity but with higher nonlinearity over a wide pressure range. Gratifyingly, the two pressure sensors were operated up to $5 \mathrm{MPa}$ with an acceptable linearity. In order to evaluate the performance of the silicon pressure sensors, the main characteristics of tested devices are presented in Table 1 , and the comparison among tested devices and the other pressure sensor is presented in Table 2. As can be seen, the increase of ambient temperature leads to the decrease of the full-scale output and sensitivity of the pressure sensor, and the increase of its nonlinearity. The micro sensor is designed for harsh applications; therefore, the thickness of the sensing diaphragm is increased to enhance the performance under high pressure. Note that the increase of diaphragm thickness leads to decreased full-scale output, nonlinearity, and sensitivity of the pressure sensor.

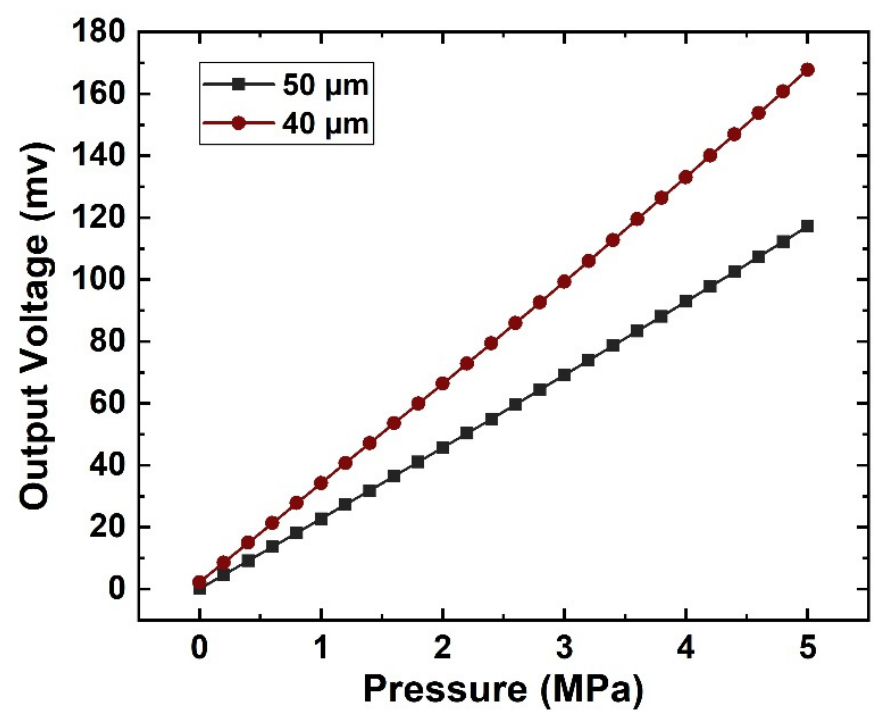

Figure 6. Output voltage of the sensor with different diaphragm thickness.

Table 1. Main characteristics of the tested devices under different test conditions.

\begin{tabular}{cccccc}
\hline Parameters & Units & \multicolumn{4}{c}{ Tested Devices } \\
\hline Operation & ${ }^{\circ} \mathrm{C}$ & 25 & 125 & 25 & 25 \\
Temperature & & & & & 50 \\
Thickness of & $\mu \mathrm{m}$ & 50 & 50 & 40 & 5.3 \\
Diaphragm & $\mathrm{V}$ & 3.3 & 3.3 & 3.3 & $0-5$ \\
Excitation Voltage & $\mathrm{MPa}$ & $0-3$ & $0-3$ & $0-5$ & 117.1 \\
Pressure Range & $\mathrm{mv}$ & 69.28 & 55.23 & 167.87 & 23.55 \\
Full-Scale Output & $\mathrm{mv} / \mathrm{MPa}$ & 22.99 & 18.52 & 33.04 & 0.41 \\
Sensitivity & F.S. $\%$ & 0.25 & 0.31 & 0.48 & 0.41 \\
Nonlinearity & & & &
\end{tabular}


Table 2. Comparison among our devices, a commercial device, and others described in literature.

\begin{tabular}{|c|c|c|c|c|c|c|}
\hline \multirow{2}{*}{$\begin{array}{l}\text { Parameters } \\
\begin{array}{l}\text { Thickness of } \\
\text { Diaphragm }\end{array}\end{array}$} & \multirow{2}{*}{$\begin{array}{c}\text { Units } \\
\mu \mathrm{m}\end{array}$} & \multicolumn{2}{|c|}{$\begin{array}{l}\text { Fabricated } \\
\text { Devices }\end{array}$} & \multirow{2}{*}{$\begin{array}{c}\text { MSD700-ASO }^{1} \\
-\end{array}$} & \multirow{2}{*}{$\begin{array}{c}\text { [14] } \\
40\end{array}$} & \multirow{2}{*}{$\begin{array}{r}{[31]} \\
1.2\end{array}$} \\
\hline & & 50 & 40 & & & \\
\hline Chip Size & $\mathrm{mm}$ & $1.5 \times 1$ & $\times 0.82$ & $0.95 \times 0.95 \times 0.6$ & $1.5 \times 1.5$ & $1.6 \times 1.6 \times 0.9$ \\
\hline Excitation Voltage & $\mathrm{V}$ & 3.3 & 3.3 & 5 & 10 & 3 \\
\hline Pressure Range & $\mathrm{MPa}$ & $0-5$ & $0-5$ & $0-0.7$ & $0-1$ & $0-0.55$ \\
\hline Full-Scale Output & $\mathrm{mv}$ & 117.1 & 167.87 & 100 & 105 & 45.99 \\
\hline Sensitivity & $\mathrm{mv} / \mathrm{V} / \mathrm{MPa}$ & 7.85 & 10.01 & 28.57 & 10.5 & 27.87 \\
\hline Nonlinearity & F.S.\% & 0.41 & 0.48 & 0.3 & - & 0.34 \\
\hline
\end{tabular}

${ }^{1}$ Product code: MSD700-ASO, MEMSensing Microsystems Co., Ltd., Suzhou, China.

\subsection{Output Characteristics under PI Protection}

The output characteristics under high pressure range of the PI protected micro pressure sensor were tested. The device with a PI protection layer was pressured from 0 to $5 \mathrm{MPa}$. In order to compare the influence of the protective layer on the output characteristics, V-P curves of the devices with and without the top surface PI layer are presented in Figure 7.

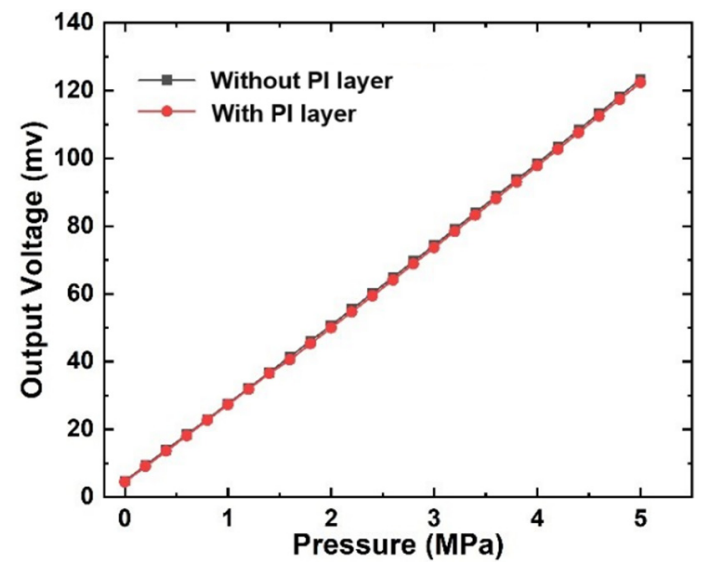

Figure 7. Output voltage of the devices with and without the PI protection layer.

The output curves show that the PI protection layer has almost no effect on the output characteristics of the micro sensor. A further experiment was designed to test the anticorrosive performance of the polyimide layer. Both devices, with and without a polyimide protection layer, were immersed in seawater and heated to $80^{\circ} \mathrm{C}$ in an oven to accelerate the corrosion of the devices. The devices were removed from the seawater and dried before a resistance test every $30 \mathrm{~min}$. Normally, the resistance of the Wheatstone bridge was between 1.7 and $2.0 \mathrm{k} \Omega$; if the output resistance was not in the normal range, the device was considered out of operation. The experiment results are shown in Table 3.

Table 3. Resistance of the Wheatstone bridge.

\begin{tabular}{ccccccc}
\hline Test Devices & Number & 0 min & 30 min & 60 min & $\mathbf{9 0 ~} \mathbf{m i n}$ & $\mathbf{1 2 0} \mathbf{m i n}$ \\
\hline Devices without polyimide & 1 & normal & open circuit & - & - & - \\
protective layer & 2 & normal & open circuit & - & - & - \\
Devices with polyimide & 3 & normal & normal & normal & normal & normal \\
protective layer & 4 & normal & normal & normal & normal & normal \\
\hline
\end{tabular}

Results show that the devices with the polyimide protective layer could still work after $2 \mathrm{~h}$ immersion in seawater, while the devices without PI layer were out of operation after $30 \mathrm{~min}$ immersion. It can be inferred that the PI protective layer possesses an exceptional anticorrosive performance. Therefore, the PI protection layer has a broad prospect on the micro pressure sensor for harsh environment applications, such as gas pressure measurement on ships or other marine engineering applications. 
In order to compare the stability of PI protection layer and traditional silicone rubber encapsulation under instantaneous violent pressure variation, a dynamic pressure test was designed. Tested pressure sensors were covered with a PI protection layer and silicone rubber, nonencapsulated sensors served as a control group. The start pressure was set at 3 , 4 , and $5 \mathrm{MPa}$. After each instantaneous decompression to normal atmospheric pressure, the output resistance was measured and compared with the control group. The process was repeated three times; if the output resistance was abnormal, the process was discontinued. Test results are presented in Table 4.

Table 4. Encapsulation stability tests.

\begin{tabular}{|c|c|c|c|c|c|}
\hline Test Cycle & Protective Layer & Pressure & 1 & 2 & 3 \\
\hline \multirow{5}{*}{ Cycle 1} & \multirow{2}{*}{ Silicone rubber } & $3 \mathrm{MPa}$ & Pass & Pass & Pass \\
\hline & & $4 \mathrm{MPa}$ & Pass & Pass & Abnormal \\
\hline & \multirow{3}{*}{ Polyimide coat } & $3 \mathrm{MPa}$ & Pass & Pass & Pass \\
\hline & & $4 \mathrm{MPa}$ & Pass & Pass & Pass \\
\hline & & $5 \mathrm{MPa}$ & Pass & Pass & Pass \\
\hline \multirow{5}{*}{ Cycle 2} & \multirow{2}{*}{ Silicone rubber } & $3 \mathrm{MPa}$ & Pass & Pass & Pass \\
\hline & & $4 \mathrm{MPa}$ & Abnormal & - & - \\
\hline & \multirow{3}{*}{ Polyimide coat } & $3 \mathrm{MPa}$ & Pass & Pass & Pass \\
\hline & & $4 \mathrm{MPa}$ & Pass & Pass & Pass \\
\hline & & $5 \mathrm{MPa}$ & Pass & Pass & Pass \\
\hline
\end{tabular}

It can be concluded from the table that the polyimide coat exhibits better stability than traditional silicone rubber encapsulation. Due to less deformation under high pressure compared with silicone rubber, the PI protection layer leads to less potential for abnormal output resistance caused by a fracture of the bonding wires. In conclusion, the polyimide protection layer has reliable corrosion resistance and high stability under violent pressure variation. Thus, it has great potential for application on micro sensors designed for harsh environments and high-pressure applications.

\subsection{Temperature Compensation System}

In addition to the large temperature difference between day and night on the sea, the operating temperature of the instruments onboard also varies from moment to moment. To overcome the sensitivity drift due to the temperature variation, a practical and flexible pressure measurement system with temperature compensation is developed to calibrate the drift caused by the ambient temperature variation. The design purpose of the embedded digital compensation system is to simplify the complexity and reduce the cost to satisfy the requirement of marine engineering. Hardware of the compensation system consists of a commercial monolithic-integrated 24-bit analog-digital convertor and signal amplifier, a high-precision temperature sensor, power management system, and a low-cost MCU. The system diagram is shown in Figure 8. 


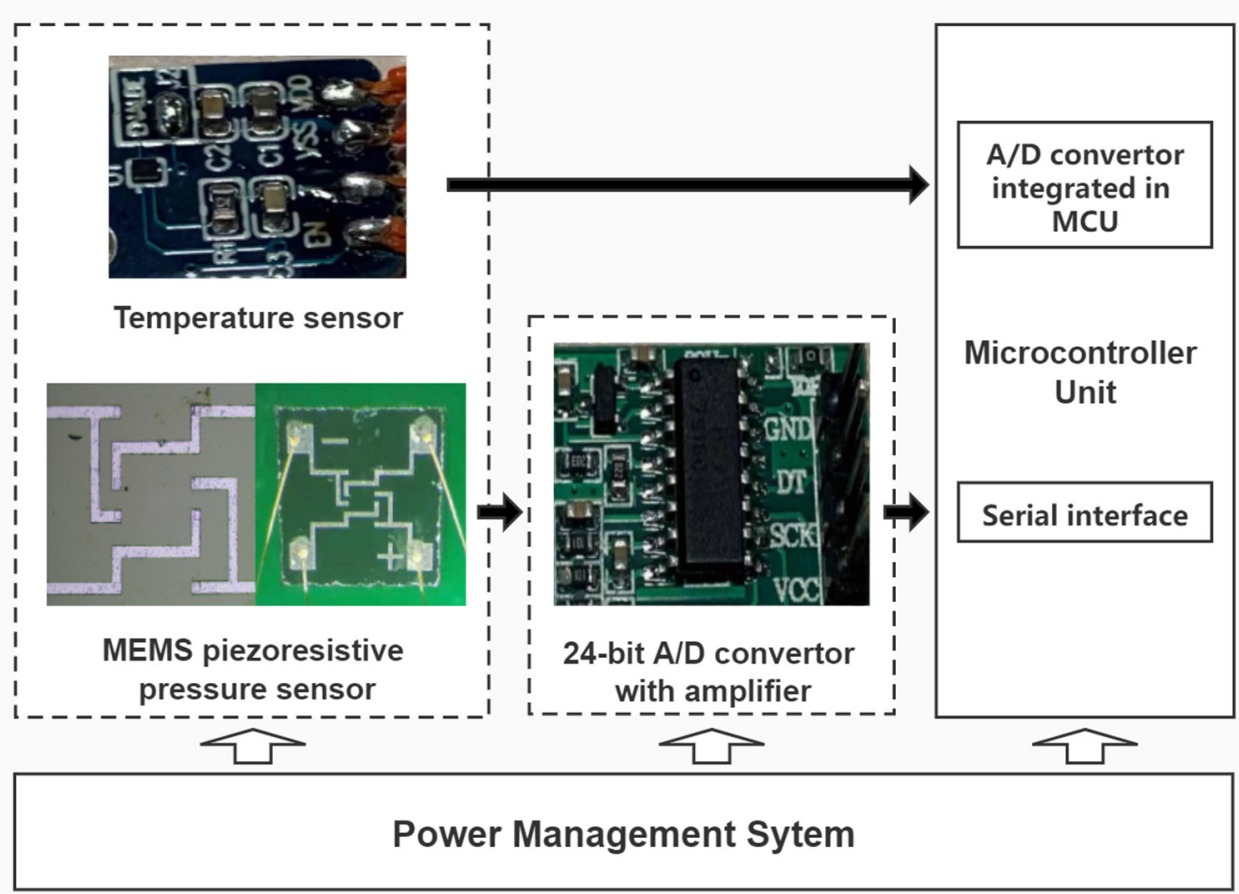

Figure 8. The diagram of the digital pressure measurement system with temperature compensation.

Generally, the output of the pressure sensor at a constant voltage can be expressed as follows:

$$
V_{\text {out }}=S_{T} \times P+V_{0}
$$

where $S_{T}$ is the sensitivity at T ${ }^{\circ} \mathrm{C}, \mathrm{P}$ denotes the incentive pressure, and $V_{0}$ represents the zero-drift voltage due to fabrication error when no incentive pressure exists.

Firstly, the zero-drift voltage $V_{0}$ is compensated by average approximation. The compensation value is obtained preliminarily by averaging the zero-drift voltages at various temperatures. After compensation, the output voltage can be described as a proportional function:

$$
V_{\mathrm{Z}}=V_{\text {out }}-\overline{V_{0}} \approx S_{T} \times P
$$

Secondly, the temperature-drift voltage is compensated by a sensitivity coefficient. After compensation, the output voltage can be described as follows:

$$
V_{\text {comp }}=K \times V_{Z} \approx K \times S_{T} \times P
$$

where $K$ denotes the compensation coefficient of $S_{T}$ The coefficient $K$ can be calculated from:

$$
K=\frac{S_{r e f}}{S_{T}}=\left\|S_{T}=a_{1} T^{2}+a_{2} T+a_{3}, S_{r e f}=22.99\right\|
$$

where $a_{1}, a_{2}$, and $a_{3}$ are the coefficients of the rational polynomial model, and $S_{\text {ref }}$ is the sensitivity at $25^{\circ} \mathrm{C}$ (which is defined as the reference). After the curve fitting of the sensitivity, the polynomial coefficients $a_{1}, a_{2}$, and $a_{3}$ were obtained: $1.657 \times 10^{-4},-0.06873$, and 24.58, respectively.

By simultaneously collecting output data from the pressure sensor and the temperature sensor through the serial ports of the microcontroller, the voltage of the pressure sensor at different temperatures can be mapped to that at the reference temperature (i.e., $25^{\circ} \mathrm{C}$ ). Therefore, the output voltage of the measurement system becomes weakly correlated with the temperature. The output characteristics of the pressure measurement system before and after compensation are shown in Figure 9a,b. The results show that the temperature 
compensation system for the pressure sensor could successfully implement the sensitivity drift compensation. The maximum error of the output value after compensation was $0.43 \%$ F.S.

(a)

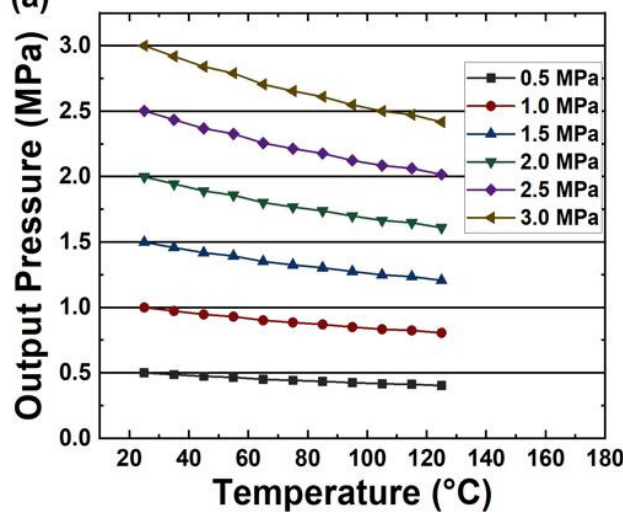

(b)

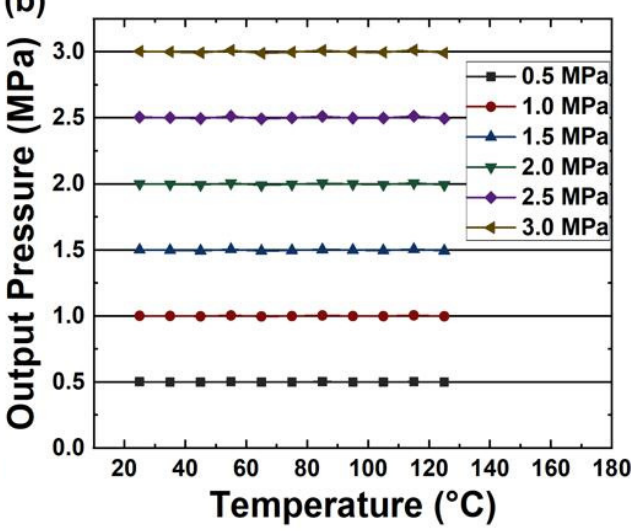

Figure 9. (a) Output without temperature compensation. (b) Output with temperature compensation.

\section{Conclusions}

Our work aimed to develop a novel solution for micro pressure sensors for harsh marine environment applications. A wide-range MEMS piezoresistive micro pressure sensor was presented, which was fabricated with the optimized bulk-micromachining technology and packaged using Si-glass bonding technology. The pressure sensors achieved reliable performance in a harsh environment such as high pressure and high temperature while using a miniature size. To manage the moist and liquid external environment for marine application, a flexible polyimide protection layer was fabricated on the top surface of the micro sensor, which showed reliable performance and stability of protection. Meanwhile, a flexible digital compensation system was developed to calibrate linearity drift in a temperature range, which showed potential for large-scale applications with low cost. However, the sensitivity of the sensor was sacrificed for measurement range and stability to some extent. In future work, we will keep focusing on structure and material optimization of piezoresistive pressure sensors to enhance the performance of pressure sensors.

Author Contributions: Conceptualization, M.W.; methodology, M.J. and M.W.; validation, M.J., B.G. and Y.F.; writing-original draft preparation, M.J.; writing-review and editing, M.W., Y.C., B.J. and G.W.; supervision, M.W.; project administration, G.W. All authors have read and agreed to the published version of the manuscript.

Funding: This work was supported in part by the National Key R\&D Program of China (2018YFE0120000); National Natural Science Foundation of China (62104056,61771175); the Fundamental Research Funds for the Provincial Universities of Zhejiang (GK199900X001); Zhejiang Provincial Natural Science Foundation of China (LQ21F010010); Zhejiang Provincial Key Research \& Development Project (2019C04003); Wenzhou Major Science and Technology Innovation Project (ZG2020016) China; Postdoctoral Science Foundation (2020TQ0246, 2021M692638); Shanghai Sailing Program (21YF1451000); and the Fundamental Research Funds for the Central Universities (31020200QD013).

Data Availability Statement: Not applicable.

Acknowledgments: The authors would like to thank the Institute of Flexible Electronics Technology of THU, Zhejiang. The authors are also grateful to Suzhou Institute of Nana-Tech and Nano-Bionics, Chinese Academy of Science.

Conflicts of Interest: The authors declare that they have no known competing financial interests or personal relationships. 


\section{References}

1. Fiorillo, A.S.; Critello, C.D.; Pullano, A.S. Theory, technology and applications of piezoresistive sensors: A review. Sens. Actuators A Phys. 2018, 281, 156-175. [CrossRef]

2. Barlian, A.A.; Park, W.-T.; Mallon, J.R.; Rastegar, A.J.; Pruitt, B.L. Semiconductor piezoresistance for microsystems. Proc. IEEE 2009, 97, 513-552. [CrossRef] [PubMed]

3. Balavalad, K.B.; Sheeparamatti, B.G. Design, simulation \& analysis of SOI based micro piezoresistive pressure sensor for high temperature applications. In Proceedings of the 2018 3rd IEEE International Conference on Recent Trends in Electronics, Information \& Communication Technology (RTEICT), Bangalore, India, 18-19 May 2018; IEEE: Bangalore, India, 2018; pp. 2163-2167. [CrossRef]

4. Tian, B.; Zhao, Y.; Jiang, Z.; Zhang, L.; Liao, N.; Liu, Y.; Meng, C. Fabrication and structural design of micro pressure sensors for tire pressure measurement systems (TPMS). Sensors 2009, 9, 1382. [CrossRef]

5. Fragiacomo, G.; Reck, K.; Lorenzen, L.; Thomsen, E.V. Novel designs for application specific MEMS pressure sensors. Sensors 2010, 10, 9541. [CrossRef]

6. Mitrakos, V.; Hands, P.J.W.; Cummins, G.; Macintyre, L.; Denison, F.C.; Flynn, D.; Desmulliez, M.P.Y. Nanocomposite-based microstructured piezoresistive pressure sensors for low-pressure measurement range. Micromachines 2018, 9, 43. [CrossRef] [PubMed]

7. Jiang, H.; Yufei, Z.; Runhui, Z.; Lirong, M.; Tao, C.; Wenjie, M.; Caofeng, P. Recent advances of wearable and flexible piezoresistivity pressure sensor devices and its future prospects. J. Mater. 2020, 6, 86-101. [CrossRef]

8. Yi, Z.; Yang, B.; Zhang, W.; Wu, Y.; Liu, J. Batteryless tire pressure real-time monitoring system driven by an ultralow frequency piezoelectric rotational energy harvester. IEEE Trans. Ind. Electron. 2021, 68, 3192-3201. [CrossRef]

9. Clementine, M.B.; Yukitoshi, K.; Bob, C.S.; Alex, C.; Anaïs, L.; Zhen, W.; James, C.; Paige, F.; Zhenan, B. A stretchable and biodegradable strain and pressure sensor for orthopaedic application. Nat. Electron. 2018, 1, 314-321. [CrossRef]

10. Liu, X.; Wei, Y.; Qiu, Y. Advanced flexible skin-like pressure and strain sensors for human health monitoring. Micromachines 2021, 12, 695. [CrossRef]

11. Tan, Y.; Ivanov, K.; Mei, Z.; Li, H.; Li, H.; Lubich, L.; Wang, C.; Wang, L. A soft wearable and fully-textile piezoresistive sensor for plantar pressure capturing. Micromachines 2021, 12, 110. [CrossRef]

12. Jian, D.; Zhijian, L.; Heng, J.; Li, S. Monolithic-integrated piezoresistive MEMS accelerometer pressure sensor with glass-siliconglass sandwich structure. Microsyst. Technol. 2017, 23, 1563-1574. [CrossRef]

13. Nag, M.; Singh, J.; Kumar, A.; Singh, K. A high sensitive graphene piezoresistive MEMS pressure sensor by integration of rod beams in silicon diaphragm for low pressure measurement application. Microsyst. Technol. 2020, 26, 2971-2976. [CrossRef]

14. Sheeparamatti, B.G.; Balavalad, K.B. Fabrication and characterization of polysilicon-on-insulator (PolySOI) and a-SOI based micro piezoresistive pressure sensor for harsh environment applications. Microsyst. Technol. 2019, 25, 4119-4133. [CrossRef]

15. Alcheikh, N.; Coutier, C.; Giroud, S.; Poulain, C.; Rey, P. Characterization and modeling of a piezoresistive three-axial force micro sensor. Sens. Actuators A Phys. 2013, 201, 188-192. [CrossRef]

16. Yozo, K.; Akio, Y. Optimum design considerations for silicon piezoresistive pressure sensors. Sens. Actuators A Phys. 1997, 62, 539-542. [CrossRef]

17. Song, P.; Si, C.; Zhang, M.; Zhao, Y.; He, Y.; Liu, W.; Wang, X. A novel piezoresistive MEMS Pressure sensors based on temporary bonding technology. Sensors 2020, 20, 337. [CrossRef] [PubMed]

18. Jinjin, L.; Qing, W.; Xu, Z.; Yao, L. Flexible strain sensor with good durability and anti-corrosion property based on metal/polymer composite films embedded with silver nanowires. Arch. Civ. Mech. Eng. 2020, 20, 133. [CrossRef]

19. Won, J.; Choa, S.H.; Yulong, Z. An integrated sensor for pressure, temperature, and relative humidity based on MEMS technology. J. Mech. Sci. Technol. 2006, 20, 505-512. [CrossRef]

20. Aryafar, M.; Hamedi, M.; Ganjeh, M.M. A novel temperature compensated piezoresistive pressure sensor. Measurement 2015, 63, 25-29. [CrossRef]

21. Peng, K.H.; Uang, C.M.; Chang, Y.M. The temperature compensation of the silicon piezo-resistive pressure sensor using the half-bridge technique. Int. Soc. Opt. Eng. 2003, 5343, 292-301. [CrossRef]

22. Yang, C.; Li, C.; Zhang, C. The application of RBF neural network in the compensation for temperature drift of the silicon pressure sensor. In Proceedings of the 2010 International Conference on Computer Design and Applications, Qinhuangdao, China, 25-27 June 2010; IEEE: Qinhuangdao, China, 2010. [CrossRef]

23. Zhou, G.; Zhao, Y.; Guo, F.; Xu, W. A smart high accuracy silicon piezoresistive pressure sensor temperature compensation system. Sensors 2014, 14, 12174-12190. [CrossRef] [PubMed]

24. Kumar, S.S.; Pant, B.D. Design principles and considerations for the 'ideal' silicon piezoresistive pressure sensor: A focused review. Microsyst. Technol. 2014, 20, 2303. [CrossRef]

25. Byunghoon, B.; Bruce, R.F.; Kyihwan, P.; Mark, A.S. Design optimization of a piezoresistive pressure sensor considering the output signal-to-noise ratio. J. Micromech. Microeng. 2004, 14, 1597-1607. [CrossRef]

26. Nallathambi, A.; Shanmuganantham, T.; Sindhanaiselvi, D. Design and analysis of MEMS based Piezoresistive pressure sensor for sensitivity enhancement. Mater. Today Proc. 2018, 5, 1897-1903. [CrossRef]

27. Song, P.; Ma, Z.; Ma, J.; Yang, L.; Wei, J.; Zhao, Y.; Zhang, M.; Yang, F.; Wang, X. Recent progress of miniature MEMS pressure sensors. Micromachines 2020, 11, 56. [CrossRef] [PubMed] 
28. Sujit, E.S.; Kusuma, N.; Hemalatha, B. Polysilicon piezoresistive MEMS pressure sensor: Study of analytical solutions for diaphragm and design \& simulation. In 2017 International Conference on Communication and Signal Processing (ICCSP); IEEE: Chennai, India, 2017; pp. 1606-1610. [CrossRef]

29. Haisheng, S.; Hong, Z.; Qiang, Z.; Yuxi, Y.; Xuyuan, C. Silicon-glass-based single piezoresistive pressure sensors for harsh environment applications. J. Micromech. Microeng. 2013, 23, 075020. [CrossRef]

30. Li, C.; Cordovilla, F.; Jagdheesh, R.; Ocaña, J.L. Design optimization and fabrication of a novel structural SOI piezoresistive pressure sensor with high accuracy. Sensors 2018, 18, 439. [CrossRef]

31. Asadnia, M.; Kottapalli, A.G.P.; Shen, Z.; Miao, J.M.; Barbastathis, G.; Triantafyllou, M.S. Flexible, zero powered, piezoelectric MEMS pressure sensor arrays for fish-like passive underwater sensing in marine vehicles. In 2013 IEEE 26th International Conference on Micro Electro Mechanical Systems (MEMS); IEEE: Taipei, Taiwan, 2013; pp. 126-129. [CrossRef]

32. Wei, C.; Zhou, W.; Wang, Q.; Xia, X.; Li, X. TPMS (tire-pressure monitoring system) sensors: Monolithic integration of surfacemicromachined piezoresistive pressure sensor and self-testable accelerometer. Microelectron. Eng. 2012, 91, 167-173. [CrossRef] 